\title{
A PDA BASED POINT OF CARE E-HEALTH SOLUTION FOR AMBULATORY CARE
}

\author{
Daniel Walsh, Carole Alcock, Lois Burgess \\ University of Wollongong \\ Joan Cooper \\ Flinders University
}

\begin{abstract}
The adoption of PDAs and mobile communication is expected to provide a solution to the use of computer technology by healthcare workers at the point-of-care. The Australian National Health Information Strategy, Health Online, is providing national leadership for approaches to address the quality and availability of information to assist in the planning and delivery of care. One area for potential growth is the availability and capture of information at the point of care by healthcare providers. A key factor in the lack of adoption of systems, is that traditionally health care information systems have been designed for desktop computing whereas many healthcare workers are highly mobile. This paper discusses phase one of a larger, four-phase project which aims to develop information access applications at point-of-care for Ambulatory Care Services. The initial phase of the research (phase one) involves workflow analysis, requirements specification and the development and testing of a system prototype to assess the feasibility of achieving increased efficiencies in workflow at the Ambulatory Care Service.
\end{abstract}

Keywords: PDAs, e-Health, mobile communications, workflow analysis, requirements specifications, prototypes

\section{INTRODUCTION}

Effective healthcare delivery within community based health services depends on efficient information access (NSW Health Information Management and IT Strategic Plan, 2001). The aim of this is paper is to present work in progress comprising the initial phase of a larger, four phase project to develop and evaluate a point-of care application for ambulatory care services to facilitate more effective delivery of healthcare. The full project involves the design and development of a personal digital assistant (PDA) based delivery system that utilises generic, reusable and scalable components. A PDA based point-of-care system is significant in that it provides for collection, delivery and exchange of timely information (both text and images) at the point-of-care, leading to a more efficient health care system. A more efficient system will overcome identified issues with existing systems by reducing the number of errors in transferring data between healthcare professionals, reducing the time spent entering and recording data (American Health Consultant, 2002) and providing for greater reliability, robustness and ease of use.

The objectives of the four phase project are:

- Production of detailed workflow diagrams through analysis of workflows in Ambulatory Care Services (Illawarra Ambulatory Care).

- Knowledge of, and solutions to, work practice issues associated with the implementation of PDAs in mobile ${ }^{22}$ healthcare situations.

${ }^{22}$ Mobile healthcare refers to any healthcare worker working within the community providing healthcare services outside the Hospital or clinical setting, eg in the home. 
- Development of a suite of middleware applications for interface software suitable for point-of-care access to databases, knowledge-repositories and directories.

- Development of generic, scalable and re-usable object oriented software components that are easily transferable to similar healthcare applications and compatible with $\mathrm{CHIME}^{23}$ (Community Health Information Management Enterprise) (Britt \& Miller, 1997).

- Development of a security, authentication and privacy framework (including policies and procedures) for ambulatory care information systems (ACIS).

- Demonstrate that computer-based information (both text and images) capture and retrieval can be integrated into point-of-care practice.

- Integration of work practice issues, scalable software and security protocols into a successful prototype PDA based healthcare delivery system.

- Provide accurate, relevant and timely access to patient management information by GPs.

Current systems at Ambulatory Care are paper based and are limited to what the healthcare worker can effectively carry. The proposed system will provide for collection, delivery and exchange of timely information at the point of care. The key advantages of the system will be its high mobility and flexibility in matching complex healthcare workflow requirements as well as immediate updating of healthcare records. Such a system has the potential to save people's lives or at least significantly improve their health outcomes by responding more quickly and with more appropriate action. This will result in better outcomes for the patient and the healthcare provider in terms of improved healthcare, better workload management and greater efficiency and effectiveness.

This paper presents the results of a prototype pilot that was developed from an initial workflow analysis and requirements specification. The pilot was developed to ascertain whether the efficiencies identified from the workflow analysis stage of the project and requirements specifications could be achieved through the use of mobile technologies deployed at the point of care.

\section{BACKGROUND}

Information systems for healthcare have been available for some decades. However, the lack of the adoption of such systems by healthcare workers around the world has limited the realisation of benefits of health information and communication technologies (ICT). It leaves healthcare exposed to problems associated with paper-based exchanges, handwriting, lack of integration of information and limited availability and capture of information at the point-of-care (Soar and $\mathrm{Yu}, 2002$ ). A key factor in the lack of adoption of these systems is that they are normally designed for desk-based users, whereas, many healthcare workers are highly mobile (Burgess and Cooper, 2001). The new technologies of hand-held devices (note-books, PDAs and tablets), Bluetooth, 802.11 wireless and broadband CDMA/3G are changing this. According to Versel (2002), PDAs appear to be

\footnotetext{
${ }^{23}$ CHIME is the information management system being adopted by NSW Health.
} 
the driving force behind the increased rate of adoption of ICT by healthcare workers. There is however, a multiplicity of issues that need to be resolved before healthcare can take full advantage of these innovations. Issues that need addressing include the development of models and principles aimed at advancing the knowledge base for mobile healthcare services generally, as well as specific software application solutions.

\section{RESEARCH APPROACH}

The research was conducted in four stages: workflow analysis, requirements specification, prototype development and testing and requirements specifications and workflow refinement.

The objectives were:

- To gain a complete understanding of the current workflows of the Illawarra Ambulatory Care Unit

- To construct workflow models that represent the activities of the Ambulatory Care Service

- To analyse the current workflows of the Service to identify the initial requirements of the Ambulatory Care PDA system prototype

- To identify problematic or inefficient areas in the workflow

- To develop and test a prototype

- To refine workflow and requirements specification

\section{Workflow Analysis}

If a transition to a mobile technology based system is to be successful, an analysis of existing workflows is an essential element. Consequently, objectives in this phase of the research are workflow focused.

Workflow analysis provided for successful definition of user needs and requirements (Kirwan \& Ainsworth (1992). Task analysis was also used to model the general activity of the ambulatory care staff at work, the processes of their interaction with the system and the processes occurring in the whole socio-technical system (Mancini et al, 2002). During workflow and task analysis, special attention was given to the wide range of worker perspectives and expectations, to issues such as patient registration, clinical records and notes, patient care plans, and access to reports, patient correspondence, appointment management and communications. Analysis of these elements was essential to maximise the value of the prototype system design. The analysis used in this study was based on observation, in-depth interviews and examination of existing workflow documents.

Workflows are closely tied to requirements. Kirwan \& Ainsworth (1992) argue that workflow analysis is a key method for the successful definition of user needs and requirements. The importance of workflow analysis is emphasised by Lowry (1997), who asserts that workflows impact on the design, which, in turn, impacts the workflows, and requirements are incorporated within these workflows. Consequently, the execution of the workflow analysis of the Illawarra Ambulatory Care Service played a pivotal role in the construction of a requirements specification for the prototype. The workflow analysis identified a number of areas of operational inefficiencies in the task set comprising the workflow model. Major tasks identified include processes related to 1) patient admission, 2) pre-patient visit, 3) patient visit, 4) post patient visit and 5) patient separation from the service. From this set, tasks 1, 2, and 3 were mapped to the prototype. 


\section{Requirements Specification}

A number of focused interviews with Ambulatory Care staff identified the initial requirements of the Ambulatory Care point of care (POC) system during the workflow analysis stage. This enabled identification of a potential solution for the operational inefficiencies in the existing workflows. These solutions form a vital part of the requirements specifications, defining important functional requirements of the prototype. In addition, staff at Ambulatory Care have identified a number of additional specific requirements they believe to be critical to the successful implementation of the POC system. An initial requirement list was developed from the workflow analysis and discussed with ambulatory care staff for verification. An analysis of the initial requirements list enabled the construction of the initial requirements specification document. This guided the development of the system prototype and provided a basis from which the final requirements specification was constructed.

\section{Development and Testing of Prototype}

The prototype was developed and implemented to identify possible errors in the requirements specification and to stimulate further feedback from Ambulatory Care staff. It is to be noted that it was not intended that the final system would be modelled on the prototype. Rather, the results from the prototype and the final requirements specification will provide a foundation from which the final system is developed. The prototype system did not fully replace existing systems, but ran in parallel; hence, there could be no accurate assessment on the systems effect on the efficiency of the entire workflow. However, by implementing the prototype system in specific areas of the workflow, system functionalities could be tested. This process allowed Ambulatory Care staff to accurately test and verify efficiency gains achieved (for individual workflow tasks: that is, tasks 1, 2, and 3), through the implementation of the prototype system.

Ambulatory Care staff were observed operating the system prototype in the three specific areas of the workflow including:

- Patient admission data-entry process

- Patient visit (point of care) data-entry process

- Patient separation data-entry process

In order to automate the three processes listed above, Hospital and Community Health databases containing patient data required by Ambulatory Care were simulated on the prototype system. This process facilitated simulation of database communication during the prototype implementation, including:

- The process of importing patient data from the hospital database during the admission process

- The uploading of patient data to the community health database during the patient admission and discharge (separation) process

- The process of recording patient notes and observations at the point of care during the patient visit

- The process of retrieving historical patient episodes to acquire patient's medical background at the point of care during the patient visit 
Observing specific workflow activities allowed mapping of prototype-integrated workflows to the paper-based data-entry workflow. Efficiency gains achieved through the implementation of the system were more accurately measured by this process. The majority of workflow areas identified as inefficient were captured in the overall data-entry process. Consequently, the majority of the requirements and functionalities identified during the workflow analysis and requirements specification stages focus on achieving efficiency gains in the data entry process. These functionalities were implemented in the prototype system to allow their impact on the Ambulatory Care Service workflow to be assessed.

\section{RESULTS AND DISCUSSION}

The initial phase of the research revealed that the implementation of a PDA based EMR system at the Ambulatory Care Service will enable more medical and patient information to be mobilised to the point of care. These findings are consistent with existing research, in particular Ammenwerth et al.(2000), Versel (2002), and Anderson (2000) who suggest that "computer based information systems can increase access to clinical information" (Anderson, 2000). This research phase also concludes that current centralised EMR systems available to the service are not used as the primary database because they do not support Ambulatory Care Staff at the point of care. The prototype implementation further revealed that the implementation of an electronic system would facilitate better communications with external medical databases, a view also held by Anderson (2000). The results of the prototype implementation also indicate that the implementation of the Ambulatory Care PDA system will support critical workflow activities, address the operational inefficiencies of the Ambulatory Care Service (by automating and streamlining workflow activities) and increase access to patient and medical information at the point of care.

A number of other benefits accrued from the prototype implementation. Firstly, achievable time saving gains through the simulation of data entry and retrievable processes. As well, additional requirements including: data entry audit controls, patient allergy reminders, data formatting standardisation, patient record search (database query), GUI design, data sharing interface, patient notes, controls and search mechanisms, usability issues, and changing and unpredictable data set. The identification of these additional requirements led to refinement of the requirement specification document, providing a more accurate reflection of the requirements of the final PDA system.

\section{CONCLUSION}

The application of existing workflow analysis methodologies resulted in the Ambulatory Care Service workflow being accurately captured, described and documented with the identification of workflow sub-tasks and the creation of workflow diagrams. The analysis of these workflow diagrams enabled the initial requirements of the Ambulatory Care prototype PDA system to be identified. Analysis of the workflow models also allowed inefficient or problematic areas of the Ambulatory Care Service workflow to be identified. Through the development and implementation of the prototype system, requirements of the final Ambulatory Care PDA system, that will assist in resolving operational inefficiencies, have now been identified. Results of this phase of the project also highlighted requirements that leverage new PDA technologies to add value to current workflows, specific requirements aimed at overcoming the limitations of paper based medical record systems, and functional requirements of the Ambulatory Care Service. Future phases of this project 
will build on this foundation research to achieve a high quality point of care eHealth application for Ambulatory Care.

\section{REFERENCES}

American Health Consultant 'PDA's Help Nurses Improve Care and Save Time: Devices Previously Used Only by Physicians" Healthcare Benchmarks and Quality Improvement, October, 2002:V.9(10): 44(3)

Ammenworth, W. et al. Mobile Information and Communication Tools in the Hospital. International Journal of Medical Informatics. 57 (2000):21-40.

Anderson, J. Computer-Based Ambulatory Care Information Systems: Recent development. Journal of Ambulatory Care Management. 23.2 (2000):53-60.

Britt, H and Miller, G “ Recent Developments in Information Management for Primary and Community Health Services” Health Information Management 1997 v26 (4): 193197

Burgess, L. and Cooper, J (2001) “ The Potential of WAP in Mobile Healthcare Delivery in Rural and Remote Communities in Australia” E-Business Review Vol. 1 Journal of the International Academy of E-Business.

Kirwan, B and Ainsworth, L (1992) “A Guide to Task Analysis” Taylor and Francis, London

Lowry, J (1997) "Workflow Analysis" Available: http://edhs1.gsfc.nasa.gov/waisdata/workshops/ClientWk106.pdf 18 December 2002.

Mancini, G, Marti, P, Palmonari, M and Rizzo, A (2002) "Task analysis and workflow methodology for cooperative work settings"

(URL: www-sv.cict.fr/cotcos/pjs/Method....analyismethods/analysispaperMancini.htm/)

New Wales Health. Information Management Division. "Information Management and Technology Strategic Plan" December, 2001.

Soar, J and Yu, P "The Future of eHealth through next generation Wireless and Mobile Connectivity” Proceedings Health Informatics Conference, HIC2002, Melbourne, August, 2002

Versel, N. "Wave of the (Not-So-Distant) Future: Annual Healthcare It Survey Shows Rise in Technology Adoption." Modern Physician 6.11 (2002) 